\title{
Politique
}

Politique

\section{Guy Bourassa et Jacques Léveillée (sous la direction de), Le système politique de Montréal, Cahiers de l'ACFAS, 1986, 600 p.}

\section{Caroline Andrew}

Numéro 11, hiver 1987

L’État privé

URI : https://id.erudit.org/iderudit/040562ar

DOI : https://doi.org/10.7202/040562ar

Aller au sommaire du numéro

\section{Éditeur(s)}

Société québécoise de science politique

ISSN

0711-608X (imprimé)

1918-6584 (numérique)

Découvrir la revue

Citer ce compte rendu

Andrew, C. (1987). Compte rendu de [Guy Bourassa et Jacques Léveillée (sous la direction de), Le système politique de Montréal, Cahiers de l'ACFAS, 1986, 600 p.] Politique, (11), 202-205. https://doi.org/10.7202/040562ar d'utilisation que vous pouvez consulter en ligne.

https://apropos.erudit.org/fr/usagers/politique-dutilisation/ 
Guy Bourassa et Jacques Léveillée (sous la direction de), Le système politique de Montréal, Cahiers de l'ACFAS, 1986, 600 p.

C'est un moment opportun pour produire un livre sur Montréal. Avec la fin du régime Drapeau et l'élection de Jean Doré l'on assiste à une recrudescence de l'intérêt pour les questions touchant la gestion de la ville, les stratégies de développement et les options politiques qui vont être adoptées par les autorités montréalaises. Et pour bien comprendre la portée des décisions il est important d'être renseigné sur les enjeux principaux, les acteurs dominants, les structures de prise de décision. Pour ces raisons la parution du livre de Bourassa et Léveillée est particulièrement bienvenue.

L'optique générale du livre le rend utile non seulement pour le présent mais également pour l'avenir. Le livre est centré sur 
l'étude du Montréal moderne - la ville depuis qu'elle est mégalopole mais aussi depuis son déclin comme principal pôle urbain du Canada. La question centrale du livre est de déterminer quelle stratégie de développement peut être envisagée pour et par Montréal, quelles structures peuvent faciliter ce développement et quelles forces politiques vont déterminer les stratégies qui vont se réaliser. Ce n'est pas un Montréal triomphant qui sert de trame de fond pour le livre c'est plutôt la ville incertaine, aux prises avec les difficultés qu'impose la recherche d'une voie pour l'avenir.

La structure du livre se comprend à partir de cette idée. Dans la première section le contexte est donné - le développement de la ville moderne et le façonnement de l'environnement socioéconomique. Ensuite viennent deux sections sur les structures politiques - la première sur celles de la ville de Montréal et la seconde, sur celles de la Communauté urbaine. Les textes dans ces sections traitent surtout de deux thèmes; la description des structures et ensuite l'évaluation du caractère démocratique des gouvernements montréalais. La section suivante, sur les forces politiques, inclue des textes sur les idéologies, les groupes de pression et le système électoral. Enfin, les deux dernières sections touchent aux grands enjeux politiques et aux stratégies de développement auxquels est confronté Montréal.

Comme tous les recueils de textes Le système politique de Montréal est inégal - certains des textes sont meilleurs que d'autres et le choix de matériel fait en sorte que certaines des sections sont plus complètes et couvrent mieux leur sujet que d'autres. La meilleure section est celle sur les enjeux et la section la plus inégale, celle sur les forces politiques. Cette dernière comprend des bons textes - notamment celui de Jean-Pierre Collin sur les élections depuis 1970 - mais il n'y a rien sur les élus, les fonctionnaires ni vraiment sur les groupes de pression. Le texte de Marc Choko sur le capital immobilier est intéressant mais il ne traite qu'indirectement des groupes de pression et il aurait 
mieux cadré dans la section sur les enjeux. Cette dernière section comprend des bons textes sur la rénovation urbaine (de Francine Dansereau), sur la réhabilitation de l'habitat (de Richard Morin), sur le transport (de Pierre Delorme) et sur les stratégies de réponse de la ville à la crise de croissance (de Jacques Léveillée). Ce dernier texte est particulièrement intéressant car il fait très bien le lien entre, d'une part, les structures et activités du gouvernement local et, d'autre part, le développement socio-économique de la ville.

En fait la force inégale des sections reflète bien l'état de la recherche sur Montréal. L'étude des processus du développement de la ville est plus avancée que celle du fonctionnement des institutions politiques. Ceci est peut-être dû en partie au caractère très fermé de l'administration municipale de Jean Drapeau mais c'est aussi lié au fait que le phénomène urbain a intéressé plus les sociologues et les économistes que les politicologues. Et, comme résultat, nous connaissons plus sur les processus de développement que sur le fonctionnement des institutions. Ce qu'il faut souhaiter c'est la continuation des recherches comme celle de Jacques Léveillée sur les stratégies de réponse qui situe les acteurs locaux par rapport aux processus de développement.

C'est une œuvre utile qu'ont fait Guy Bourassa et Jacques Léveillée. La parution d'un recueil comme celui-ci peut contribuer à stimuler l'intérêt pour l'étude de la vie politique montréalaise. Constater non seulement la richesse de certaines analyses, mais aussi certaines lacunes peut susciter de nouvelles études dans les domaines qui n'ont pas été suffisamment examinés. On peut espérer en particulier que les fonctionnaires, les élus et les groupes de pression seront l'objet d'études plus approfondies afin que la deuxième édition de ce recueil puisse mieux refléter la centralité de ces acteurs pour une compréhension de la vie politique montréalaise. Et, sur la base de ces recherches, il sera possible de mieux comprendre le rôle de la ville de Montréal et de la CUM 
dans le développement socio-économique de la métropole. C'est un ambitieux programme de recherche mais la publication de ce recueil est un pas dans la bonne direction et nous $\mathrm{y}$ incite.

Caroline Andrew

Université d'Ottawa 